\title{
Polarized absorption spectra of highly oriented two-dimensional aggregates of tetrachlorobenzimidazolocarbocyanine in thin films
}

\author{
Serdar Özçelik ${ }^{\mathrm{a}, *}$, Demet Gülen ${ }^{\mathrm{b}}$ \\ ${ }^{a}$ Chemistry Department, İmir Institute of Technology, Urla 35430, İmir, Turkey \\ ${ }^{\mathrm{b}}$ Physics Department, Middle East Technical University (METU), 06531 Ankara, Turkey
}

Available online 21 December 2007

\begin{abstract}
Reaching a control on the mesoscopic morphology and internal molecular arrangement of cyanine aggregates is an important step for realization of devices with tailor-made optical properties. Despite a wealth of research, understanding of the relationship between molecular organization, excitonic states and dynamics of aggregates is still preliminary. To this end, we have employed polarized absorption spectroscopy to investigate the relationship between internal molecular organization and excitonic states of J-aggregates in 1,1',3,3'tetraethyl-5,5',6,6'-tetrachlorobenzimidazolocarbocyanine (TTBC) thin films in poly-vinyl alcohol (PVA). Angular dependence of the UV-vis spectra has been measured at 11 different orientations between the electric field polarization and the macroscopic alignment axis. Aggregate spectral response consisted of an asymmetrically split Davydov pair of bands exhibiting opposite polarization: an H-band (505 nm, Lorentzian-like, polarized along the macroscopic film axis) and a J-band (594 nm, one-dimensional J-aggregate like band shape, polarized perpendicular to the macroscopic film axis). The polarized absorption observations were found to be consistent with a herringbone model for which the internal molecular arrangement, the excited state structure and dynamics have recently been detailed by us upon interpretation of isotropic absorption data in ionic aqueous solution.
\end{abstract}

(C) 2007 Elsevier B.V. All rights reserved.

Keywords: Cyanines; J-aggregates; Frenkel exciton; Herringbone arrangement; Polarized UV-vis absorption; Vertical spin coating

\section{Introduction}

Cyanines are organic molecules that can be self-assembled into ordered molecular aggregates [1]. As such they offer nanoscale molecular assemblies, which exhibit interesting excitonic properties and continue to attract attention, as for example imaging probes, artificial light-harvesting complexes and electronic energy transport wires [1-3].

The lowest energy level of J-aggregates (J-band) is a strong red-shifted superstate that is strongly polarized. It is, therefore, of particular importance for potential applications to characterize thin films in which the aggregates are aligned preferentially. Of the techniques, the vertical spin coating method (VSCM) [4] is well known to produce thin films consisting of preferentially oriented aggregates. Furthermore, the VSCM is suggested to produce films durable at room temperature in which the

\footnotetext{
*Corresponding author. Tel.: + 902327507557 ; fax: +902327506125.

E-mail address: serdarozcelik@iyte.edu.tr (S. Özçelik).
}

preferential alignment of the long J-aggregates is in the film growth direction [4]. Detection of the incident polarization dependence of spectral response of the aggregates with a well-defined macroscopic alignment direction can be very advantageous for determination of their internal molecular arrangement [3].

To this end, we investigated the structure-spectroscopy -function relationship of the tetrachlorobenzimidazolocarbocyanine (TTBC) aggregates in (poly-vinyl alcohol) PVA thin films prepared by the VSCM employing polarized absorption spectroscopy, and discussed the experimental results within a model that we have recently proposed for the internal molecular packing and the structure and dynamics of excited states of TTBC aggregates [5].

\section{Experimental methods}

TTBC (Hayashibara, Japan) was used without further purification. PVA (Aldrich, USA) was used as received. 
Thin films of aggregates dispersed in PVA films were prepared by the VSCM [4]. The spectra were recorded by an HP Agilent 8543 spectrophotometer (resolution of $0.5 \mathrm{~nm}$ ) at room temperature. The electric field polarization of the excitation was adjusted by inserting a linear glass polarizer (100:1) between the slit of the spectrophotometer and the sample. The macroscopic alignment axis of the film was adjusted to the incident polarization direction (parallel to the $0-180^{\circ}$ axis of the stage holding the sample). The stage was rotated around $\left(\approx 10^{\circ}\right)$ an axis perpendicular to the substrate surface until absorbance around $500 \mathrm{~nm}$ reached its maximum. The angle between the incident polarization and the film growth direction was step-by-step changed by rotating the stage further in the same direction at $10-20^{\circ}$.

\section{Results and discussion}

The isotropic absorption spectrum $\left(\mathrm{A}_{\mathrm{iso}}\right)$ of monomeric TTBC (Fig. 1) displays a $0-0$ transition $(\approx 514 \mathrm{~nm})$ and a vibrational $(0-1)$ side band $(\approx 480 \mathrm{~nm})$. Fig. 1 also compares the polarized absorption spectrum at $\theta=20^{\circ}$ with the $\mathrm{A}_{\text {iso }}$ in ionic aqueous solution. These two spectra are very similar. The similarities are pertinent to the peak positions and the shapes/widths of the bands. We attribute

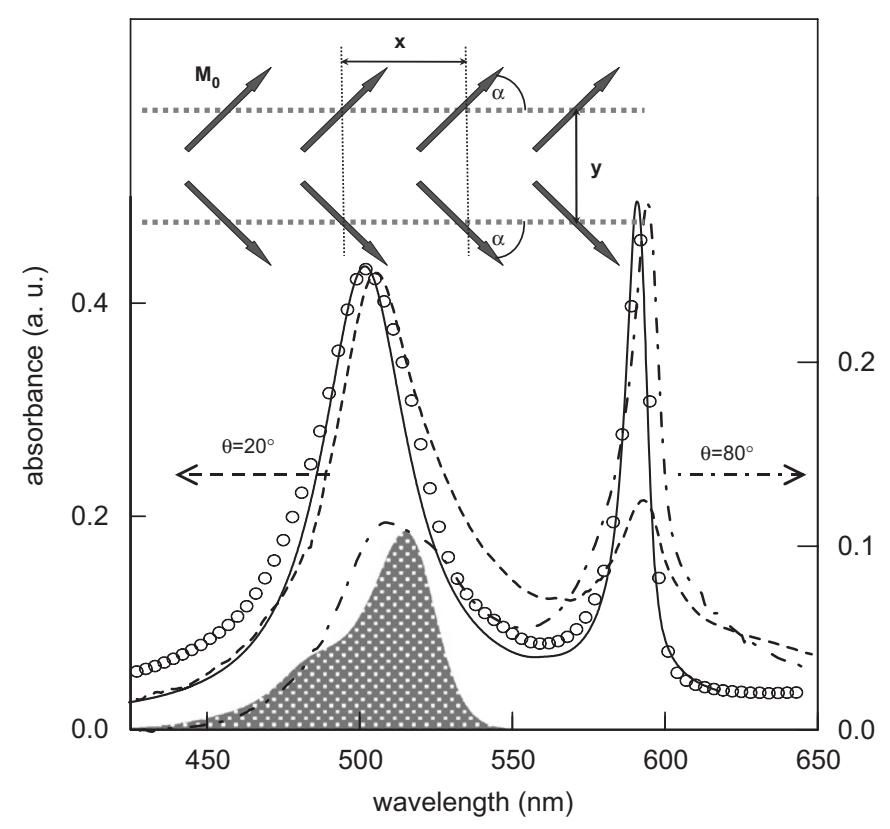

Fig. 1. Monomeric absorption spectrum in methanol (shaded), $\mathrm{A}_{\text {iso }}$ in ionic aqueous solution $(0.2 \mathrm{mM}$ TTBC in $0.01 \mathrm{M} \mathrm{NaOH}$ solution [5], symbols), the polarized absorption spectrum for $\theta=20^{\circ}$ (dash) and $\theta=80^{\circ}$ (dash-dot), simulation of $\mathrm{A}_{\text {iso }}$ (solid, taken from Ref. [5]). The polarized absorption spectra are drawn on an apparent scale because concentration of the aggregate molecules is not known and the others are scaled arbitrarily. The insert shows the herringbone model. The arrows indicate the monomeric transition dipoles of magnitude $M_{0}$. The dipole-dipole interactions facilitate the excitonic properties. For the $\mathrm{A}_{\text {iso }}$ simulation $M_{0}^{2} / x^{3}=58.40$ debye $^{2} / \mathrm{nm}^{3}, \quad M_{0}^{2} / y^{3}=339.70$ debye ${ }^{2} / \mathrm{nm}^{3}$ and $\alpha=27^{\circ}$, e.g., if $M_{0}=12$ debye, then $x=1.351 \mathrm{~nm}, y=0.7512 \mathrm{~nm}$. Here, the purpose has been to illustrate that the simulation is rather satisfactory. Please refer to Ref. [5] for further details on the simulation. the differences in the band shape/widths to some spectral heterogeneity and to the enhanced monomeric contribution on the red side of the H-band in the film. The aggregate spectra are characterized by two major bands, located asymmetrically about the $0-0$ transition and separated by a large energetic gap of $\approx 3000 \mathrm{~cm}^{-1}$ : a narrow red-shifted band $\approx 594 \mathrm{~nm}$ (the J-band) and a broad blue-shifted band $\approx 505 \mathrm{~nm}$ (the $\mathrm{H}$-band). It is well known that the band positions depend to a large extent on the internal molecular arrangement, whereas the band shapes/widths are determined basically by the excited state dynamics (see e.g., Ref. [5]). The immediate conclusion following this comparison is that TTBC aggregates assume very similar internal molecular packing and dynamics of excited states in ionic aqueous solution and in thin films.

We have already carried out a comprehensive analysis of the exciton-band structure and the dynamics of the excited states in solution [5]. This analysis was based on the simulations of the $A_{\text {iso }}$ in the Frenkel exciton formalism [5-9] and on the discussion of the fluorescence excitation and emission data. A typical simulation is shown and is detailed in Fig. 1. Strong theoretical evidence for the formation of two-dimensional aggregates assembled in herringbone arrangement was provided. The $\mathrm{H}-$ and J-bands were identified as a Davydov-split pair [6] that resulted from the coupling between the two linear J-aggregate chains of the herringbone. In addition, the wide gap between the Davydov-split bands was suggested to be bridged by the exciton band(s) of the loosely coupled chains and the monomeric species. The dynamics of the excited states were attributed to disorder-induced phononassisted intra- and interband exciton relaxations. Intraband relaxation (estimated to occur for about a picosecond in both bands) could alone account for the J-band shape/ width. The H-band shape/width was explained by further lifetime shortening of the H-band excitonic states through interband processes. Very fast interband dynamics $(\approx 50 \mathrm{fs})$ has been accounted by either direct relaxation from the $\mathrm{H}$ - to J-band or relaxation via the gap states and energy transfer to monomeric species.

Next we shall discuss that the polarized absorption spectra are compatible with the herringbone model, in which the polarizations of the $\mathrm{H}$ - and $\mathrm{J}$-bands are, respectively, along the aggregate axis and perpendicular to the aggregate axis for the homogeneous infinite aggregates [7]. These assignments for the polarization directions hold to a good approximation for the inhomogeneous finite aggregates [8].

Fig. 2 shows the absorption spectra measured at 11 different incident polarization directions between $\theta=0^{\circ}$ and $180^{\circ}$. The spectra between $\theta=0^{\circ}$ and $90^{\circ}$ are presented in comparison with the spectra between $\theta=100^{\circ}$ and $180^{\circ}$. $\mathrm{In}$ addition to the $\mathrm{H}$ - and $\mathrm{J}$-bands, some monomeric contribution, which is clearly distinguished for the $\theta=60-100^{\circ}$ spectra, is present. The Davydov-split bands whose positions are independent of the incident polarization direction exhibit opposite polarization dependence. 



Fig. 2. Polarized absorption spectra for 11 different polarization directions between $\theta=0^{\circ}$ and $180^{\circ}$. The spectra shifted down by 0.1 are drawn on the same scale as the group of spectra at $\theta=0^{\circ}, 160^{\circ}$ and $180^{\circ}$. $\theta=0^{\circ}, 20^{\circ}, 40^{\circ}$ and $60^{\circ}$ (dash); $\theta=80^{\circ}$ (dash-dot); $\theta=90^{\circ}$ (long dash); $\theta=100^{\circ}, 120^{\circ}, 140^{\circ}$ and $160^{\circ}$ (solid); and $\theta=180^{\circ}$ (dash-dot). The $\theta-$ dependence of the peak intensities of the $\mathrm{H}$ - and J-bands is shown below.

The $\theta$-dependence of the peak intensities of the $\mathrm{H}$ - and $\mathrm{J}$-bands is plotted in the inset of Fig. 2. The H-band assumes its maximum intensity when the electric field polarization and the film growth direction are close to being parallel $\left(\theta\right.$ around $0^{\circ}$, and between $160^{\circ}$ and $\left.180^{\circ}\right)$. It is observed to be strongly reduced and to assume its minimum value when the film growth axis and $E$ are close being perpendicular $\left(\theta \approx 80^{\circ}\right)$. Conversely, the J-band intensity assumes its minimum (maximum) value when the $\mathrm{H}$-band intensity attains its maximum (minimum). In between a gradual decrease (increase) in the H-band (J-band) intensity follows the changes in $\theta$. The experimentally observed symmetry pertains for polarization directions complementing at $\approx 160^{\circ}$. Under the proposition that VSCM produces films in which the aggregate's long axis lies preferentially along the film growth direction [4], the data suggest that the $\mathrm{H}$-band is polarized more or less parallel to the aggregate axis and the J-band has a polarization more or less perpendicular to it. These directions match very well with the polarizations of the $\mathrm{J}$ - and $\mathrm{H}$-bands of the herringbone aggregates.

\section{Conclusions}

On the basis of the data and the discussion provided above, we conclude that the polarized absorption spectra provide further support for the validity of herringbone arrangement, excited state structure and dynamics of the TTBC aggregates as we have recently suggested [5]. The general conclusions on the structure, dynamics and static properties of exciton bands discussed above are expected to hold for other cyanine aggregates which show the same generic features [10].

\section{Acknowledgments}

S. Ö. acknowledges TÜBİTAK-NSF Joint Grant TBAG U-12. We thank John R. Lombardi and Onur Atasoylu for their support.

\section{References}

[1] T. Kobayashi, J-Aggregates, World Scientific, Singapore, 1996.

[2] R.P. Haugland, in: J. Gregory (Ed.), Handbook of Fluorescent Probes and Research Products, ninth ed., Molecular Probes, 2002, pp. 871-873.

[3] H. Van Amerongen, R. Van Grondelle, L. Valkunas, Photosynthetic Excitons, World Scientific, Singapore, 2000.

[4] K. Misawa, H. Ono, K. Minoshima, T. Kobayashi, Appl. Phys. Lett. 63 (1993) 577.

[5] B. Birkan, D. Gülen, S. Özçelik, J. Phys. Chem. B 110 (2006) 10805.

[6] A.S. Davydov, Theory of Molecular Excitons, Plenum, New York, 1970.

[7] G. Juzeliũnas, J. Knoester, J. Chem. Phys. 112 (2000) 2325.

[8] D.M. Basko, A.N. Lobanov, A.V. Pimenov, A.G. Vitukhnovsky, Chem. Phys. Lett. 369 (2003) 192.

[9] H. Fidder, J. Knoester, D.A. Wiersma, J. Chem. Phys. 95 (1991) 7880.

[10] I.G. Scheblykin, O.Y. Sliusarenko, L.S. Lepnev, A.G. Vitukhnovsky, M. Van der Auweraer, J. Phys. Chem. B. 105 (2001) 4646. 